\title{
Influence of temperature and electrolyte concentration on regime maps in vertical-adiabatic two-phase pipe flow
}

\author{
G. Monrós-Andreu ${ }^{1}$, R. Martínez-Cuenca ${ }^{1}$, S. Torró ${ }^{1}$, \\ J. L. Muñoz-Cobo ${ }^{2} \&$ S. Chiva ${ }^{1}$ \\ ${ }^{I}$ Department of Mechanical Engineering and Construction, \\ Universitat Jaumé I, Castello de la Plana, Spain \\ ${ }^{2}$ Institute for Energy Engineering, Universitat Politècnica de València, \\ Spain
}

\begin{abstract}
The study of flow configuration and regime transitions in a vertical pipe is of primary interest for the understanding of the fundamentals of two-phase flow dynamics. Also, it is useful for the calibration of both interfacial forces and population balance models. This simple geometry allows for the isolation of each effect as the dynamics takes place mainly along one dimension. Most investigations deal with the flow configuration for different flow rates of both phases. But further investigations and new interesting results can be obtained by changing the physical properties of the base fluids and analyzing their influence on the flow dynamics. In this work we show the effects of temperature and electrolyte concentration in the flow configuration. The rise of temperature produces an unaccounted acceleration of the transitional effects. The addition of $\mathrm{NaCl}$ produces a delay of such effects thanks to the inhibition of bubble coalescence.
\end{abstract}

\section{Introduction}

Multiphase flow has been intensively studied during last decades as it occurs in a vast amount of industrial applications [1]. It takes place in both nuclear and concentrating solar power plants as they rely on the boiling of water in Rankine and/or Brayton cycles [2]. Also, industrial systems based on the mixing of 
compounds (as in chemical or pharmaceutical industries) require the study of the behaviour of two or more phases that interact between them [3]. Aeronautics also deal with the complexity of multiphase-flows as the huge changes in pressure that take place in most turbines and engines may lead to cavitating phenomena. Recent studies point out that wastewater treatment plants can be optimized by a proper analysis of the chemical compounds distribution and the aeration processes $[4,5]$.

This huge impact onto industry makes it desirable the development of simulation tools for the design and optimization of these systems. At present, these processes are being monitored by using one dimensional codes such as TRACE and RELAP, for the nuclear industry, or BioWin and SCADA for the wastewater systems. But the development of proper models for the modelling through three-dimensional transient Computational Fluid Dynamics (CFD) codes may open a new path for the optimization of these systems, unveiling the existence of inefficient designs, unexpected recirculating regions, dead zones or even sources of damage.

The existing models for the simulation of two-phase flow lack of the required generality for these purposes. Model constants need to be tuned for each specific geometry, and strong calibration is needed based on the comparison between simulation results and the actual setup that is being modelled. The development of more general models requires complete experimental databases in simple geometries. These databases serve to test the proposed models and even isolate several phenomena in order to analyze the performance of each model separately. Typically, these experiments deal with the flow configuration at several flow-rate combinations for both phases [6-9]. Other studies also deal with the influence of the diameter of the pipe on the flow configuration, as the Taylor instability impedes the formation of slugs for sufficiently big diameter pipes [10-12]. Also, some researchers have performed studies with different base fluids [13] and different gas injection methods $[14,15]$.

At present, few tools can be employed to predict the flow structure for a given gas-liquid flow rate configuration. The Taitel map [16] is one of such tools, but its generality is still a matter of debate. Also, the length that is needed in order to obtain a fully developed two-phase flow is still unknown. In this work we analyze the effects of temperature and $\mathrm{NaCl}$ concentration (from 0 up to $3500 \mathrm{ppm}$ in weight) on both the Taitel map and the pipe length that is needed to obtain a fully developed flow. Section 2 describes the experimental facility that was used. Next, the basics of the two-phase flow regimes and void profiles are introduced in Section 3. Sections 4 and 5 aim at the analysis of the effects produced but the temperature and the addition of electrolytes on the flow configuration, respectively. Finally, we summarize the main results of the work in the Conclusions.

\section{Experimental facility}

The Vertical Gas-Liquid Adiabatic Steady-State (V-GLASS) loop was used in order to acquire the experimental data. As illustrated in Figure 1, the test section consists in an upward-flow vertical pipe section with $52 \mathrm{~mm}$ inner diameter and a length of $5500 \mathrm{~mm}$. 
The gas comes from a pressurized air line that supplies a maximum pressure of 5 bar. The liquid is taken from the main water line and a purifier is used to obtain slightly contaminated water $(\sim 30 \mu \mathrm{S} / \mathrm{m})$. The clean water is stored in a 5001 tank at $20^{\circ} \mathrm{C}$ thanks to a heat exchanger (Euroklimat R407C SMART 400/30/50). Both fluids are mixed in a mixing chamber composed by 4 separate compartments, each one having a liquid inlet at the bottom and a gas inlet through a sparger that is oriented axially with the main flow. This configuration provides a quite uniform distribution of gas and liquid at the pipe inlet.

The system is named adiabatic to stress the fact that no boiling nor cavitation phenomena take place within the test section. Also, heat exchange rate is neglectable for most purposes.

The steady state conditions are ensured by using an automated control system so that the measurements take place when flowrate oscillations fall below a $5 \%$ threshold for both phases.

The local gas flow parameters were measured by using four sensor conductivity probes at three axial ports, located at $z_{b o t}=1166 \mathrm{~mm}, z_{m i d}=3176 \mathrm{~mm}$ and $z_{\text {top }}=5131 \mathrm{~mm}$ from the inlet. Note that these distances correspond to 22, 61 and 99 pipe diameters (Z/D), respectively.

Additionally, water temperature in the loop was monitorized by using PT100 thermocouples (locations T0 to T3). Note that the experimental facility also permits the measurement of liquid phase properties thanks to the Laser Doppler Anemometer. As the aim of this work deals with the gas distribution no more information on these measurements needs to be supplied.

\section{Flow regimes and the Taitel map}

For co-current upward flow gas and liquid in a vertical tube, the distribution of the liquid and gas phases changes into different recognizable flow structures or flow patterns [1]. We limit our study to bubbly or dispersed flow to transition bubblyto-slug.

The typical void profiles for dispersed flow are shown in Fig. 2. For low void conditions, the gas is attached to the wall thanks to the lift force acting onto small bubbles and a characteristic wall-peak shape is present (see wall-peak I). As the void increases, the gas starts to populate the center of the pipe thanks to the turbulent dispersion and different profiles are obtained (see wall-peaks of type IIa and IIb). For higher void fractions the gas bubbles tend to coalesce and form large bubbles that are pushed towards the pipe center by the action of the lift and a characteristic core-peak shape is obtained (core I and II distributions). This corepeak shape is characteristic in slug patterns. In between, a transitional-flat profile can be observed for some flow conditions.

We propose the following condition (Equation 1) in order to discriminate between core and wall-peaked profiles:

$$
\frac{\int_{0}^{R-D_{b}} \alpha(r) \cdot d r}{\left(R-D_{b}\right)^{2}} \leqslant \frac{\int_{R-D_{b}}^{R} \alpha(r) \cdot d r}{R^{2}-\left(R-D_{b}\right)^{2}}, \quad \text { Wall-peak condition }
$$




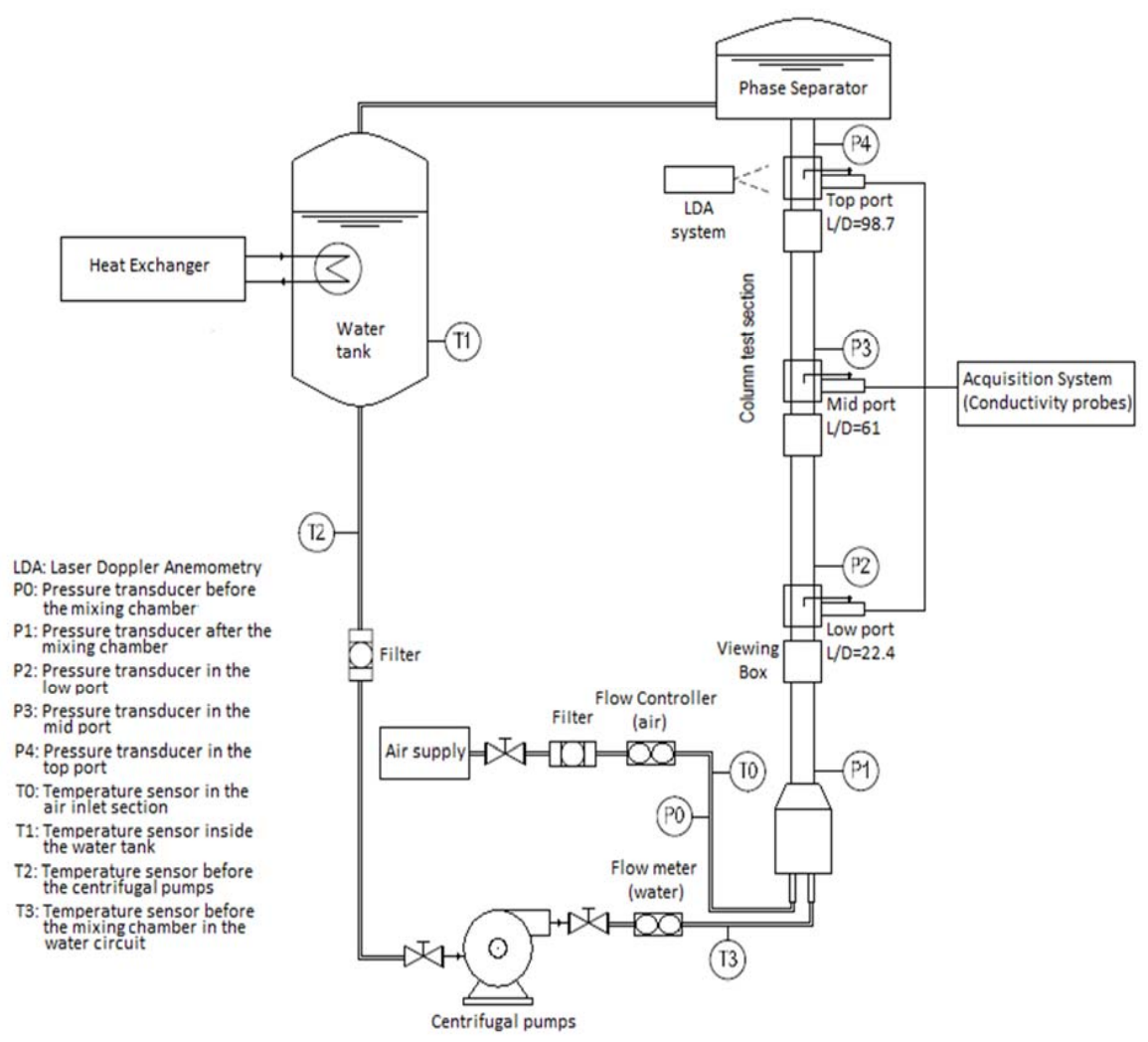

Figure 1: Sketch of the V-GLASS loop.

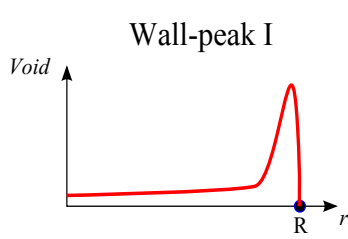

Transitional

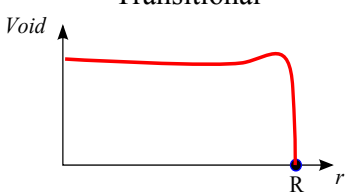

Wall-peak IIa

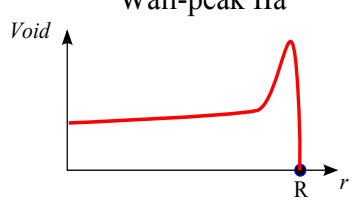

Core I

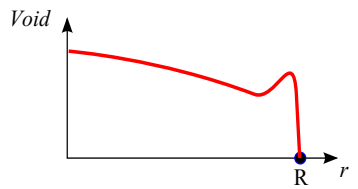

Wall-peak IIb

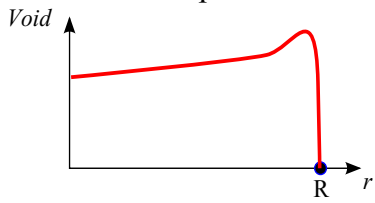

Core II

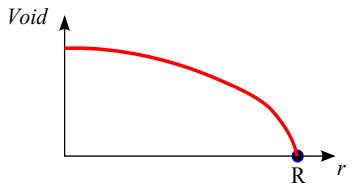

Figure 2: Typical void profiles. 


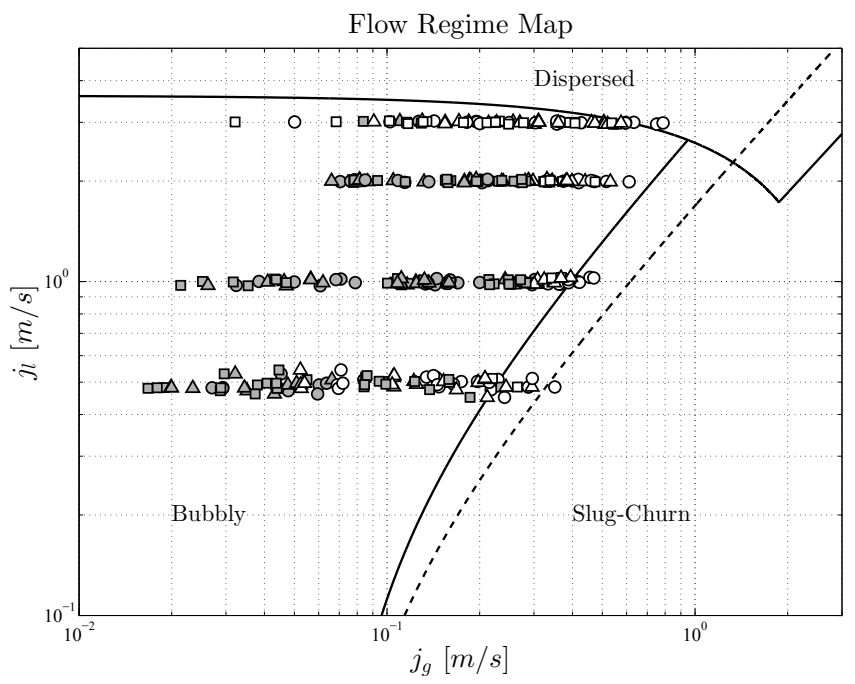

Figure 3: Taitel map for the flow conditions of the VGLASS at $20^{\circ} \mathrm{C}$ and with no electrolyte.

where $D_{b}$ is the characteristic bubble diameter or Sauter Mean Diameter, $\alpha(r)$ the void fraction at each radial position and $\mathrm{R}$ the radius of the pipe. We compare the resulting classified conditions with the correlations proposed by Taitel et al. [16] (solid line) and Kaichiro and Ishii [17] (dashed line). The resulting comparison is plotted in Fig. 3. We use filled symbols for disperse and bubbly (wall-peak) conditions end empty symbols for bubbly-to-slug transition (corepeak). Experimental data sets are performed at three different axial pipe locations, we use square symbols for experiments at $\mathrm{Z} / \mathrm{D}=22$, triangular symbols for $\mathrm{Z} / \mathrm{D}=61$ and round symbols for $\mathrm{Z} / \mathrm{D}=99$.

\section{Temperature effects}

The temperature has a strong influence on basic fluid properties such as the viscosity of the liquid phase, $\mu_{l}$, and the surface tension, $\sigma$. Viscosity mainly affects the bubble Reynolds number, $R e_{b}$, given by

$$
R e_{b}=\frac{\rho_{l} D_{b} U_{b}}{\mu_{l}},
$$

with $\rho_{l}$ the density of the liquid phase and $U_{b}$ the velocity of the bubble with respect to the liquid. The surface tension appears in the Eötvös number, E $o_{b}$, defined as:

$$
E o_{b}=\frac{\left(\rho_{l}-\rho_{g}\right) g D_{b}^{2}}{\sigma} .
$$



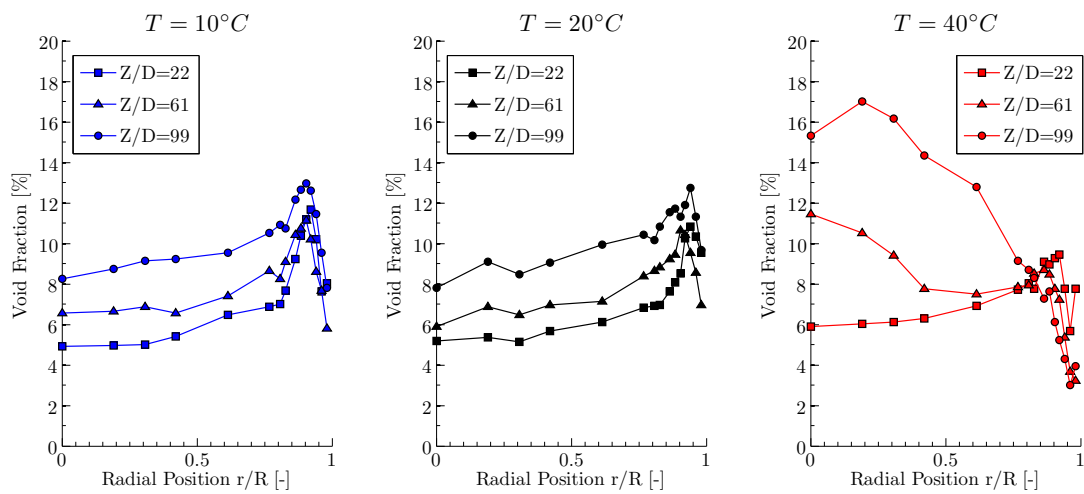

Figure 4: Axial development for the condition $j_{l}=0.5 \mathrm{~m} / \mathrm{s}$ and $j_{g}=0.05 \mathrm{~m} / \mathrm{s}$.

In this equation, $\rho_{g}$ stands for the gas density. Finally, the Morton number can be strongly affected by these changes due to its non-linear dependence on these fluid properties:

$$
M o_{b}=\frac{\left(\rho_{l}-\rho_{g}\right) g \mu_{l}^{4}}{\rho_{l}^{2} \sigma^{3}} .
$$

Note that these dimensionless groups appear in most correlations for drag and lift, so a change in their values is expected to have a strong influence in the flow dynamics [18-20].

The VGLASS permitted the acquisition of void profiles at different temperatures. Fig. 4 illustrates the axial development of the void profile across the pipe at three different temperatures for the flow condition $j_{l}=0.5 \mathrm{~m} / \mathrm{s}$ and $j_{g}=0.05 \mathrm{~m} / \mathrm{s}$. At $10^{\circ} \mathrm{C}$ the void profile at the bottom port is a wall peak of type IIa. The flow evolves up to a type IIb wall-peak at the top port. A similar trend can be noticed at $20^{\circ} \mathrm{C}$. But at $40^{\circ} \mathrm{C}$ the flow at the bottom port is of type IIb and reaches a core distribution of type II at the top port. In the mid, the flow was still in an intermediate core of type I. From these plots, it can be concluded that a rise in the temperature tends to concentrate the gas at the center of the pipe.

Fig. 5 illustrates the axial development for the flow condition $j_{l}=0.5 \mathrm{~m} / \mathrm{s}$ and $j_{g}=0.2 \mathrm{~m} / \mathrm{s}$. At $10^{\circ} \mathrm{C}$, the flow is wall-peak of type IIb at the bottom and middle locations, but core II at the top port. An increase in the temperature up to $20^{\circ} \mathrm{C}$ produces a faster axial development. Although a wall-peak of type IIb is still present in the bottom, the middle profile is already a core II distribution. Finally, at $40^{\circ} \mathrm{C}$ even the bottom distribution is a fully developed core distribution. Again, the trend of increasing temperature leading to a faster transition wall-core is observed.

This trend can be confirmed by plotting all the measured conditions in a Taitel map, see Fig. 6. Actually, the map for $10^{\circ} \mathrm{C}$ shows an accurate prediction of the transition region. But for $20^{\circ} \mathrm{C}$ the transition curve clearly deviates from the 

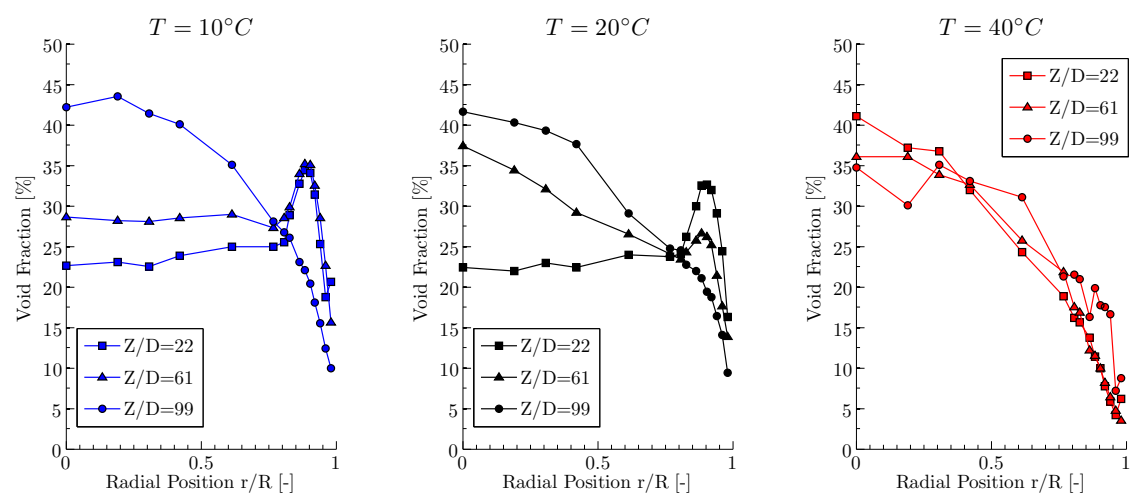

Figure 5: Axial development for the condition $j_{l}=05 \mathrm{~m} / \mathrm{s}$ and $j_{g}=0.2 \mathrm{~m} / \mathrm{s}$.

experimental data. This trend is confirmed for $40^{\circ} \mathrm{C}$, where almost no wall-peaks were obtained.

Further investigations are needed in order to verify if these effects are accounted for by present correlations based on the Reynolds, Eötvös and Morton numbers [18].

\section{Electrolyte concentration effects}

The electrolyte concentration (in case for $\mathrm{NaCl}$ and water) has little influence on the viscosity and surface tension of the working fluids [21]. But as we show in this section, it has a strong influence on the flow configuration. In this case, the flow properties were measured at 5 different electrolyte concentrations.

Fig. 7 illustrates the axial development for the flow condition $j_{l}=1 \mathrm{~m} / \mathrm{s}$ and $j_{g}=0.35 \mathrm{~m} / \mathrm{s}$ at $20^{\circ} \mathrm{C}$, the flow is wall-peak of type $\mathrm{IIb}$ at the bottom location, transitional at middle locations and fully developed core II at the top port. At the bottom port, the addition of electrolyte has little influence, although it can be noticed that the wall-peak for the case with no electrolyte has the smaller wall peak. Even for small electrolyte concentrations the most evolved profile reached at bottom and middle locations is wall-peak of type IIa, no axial development can be noticed in these cases. Only for top location, transitional or even core-I shapes (for lower electrolyte concentrations) can be observed, replacing the core II corresponding to no electrolyte case.

Fig. 8 illustrates again the core-peak delaying effect of increasing electrolyte concentration and also the flattening effect produced in the interfacial velocity profiles, produced by the decrease of bubble size which produces more homogeneous flow. Again, the trend of increasing electrolyte concentration leading to a slower transition wall-core is observed. 

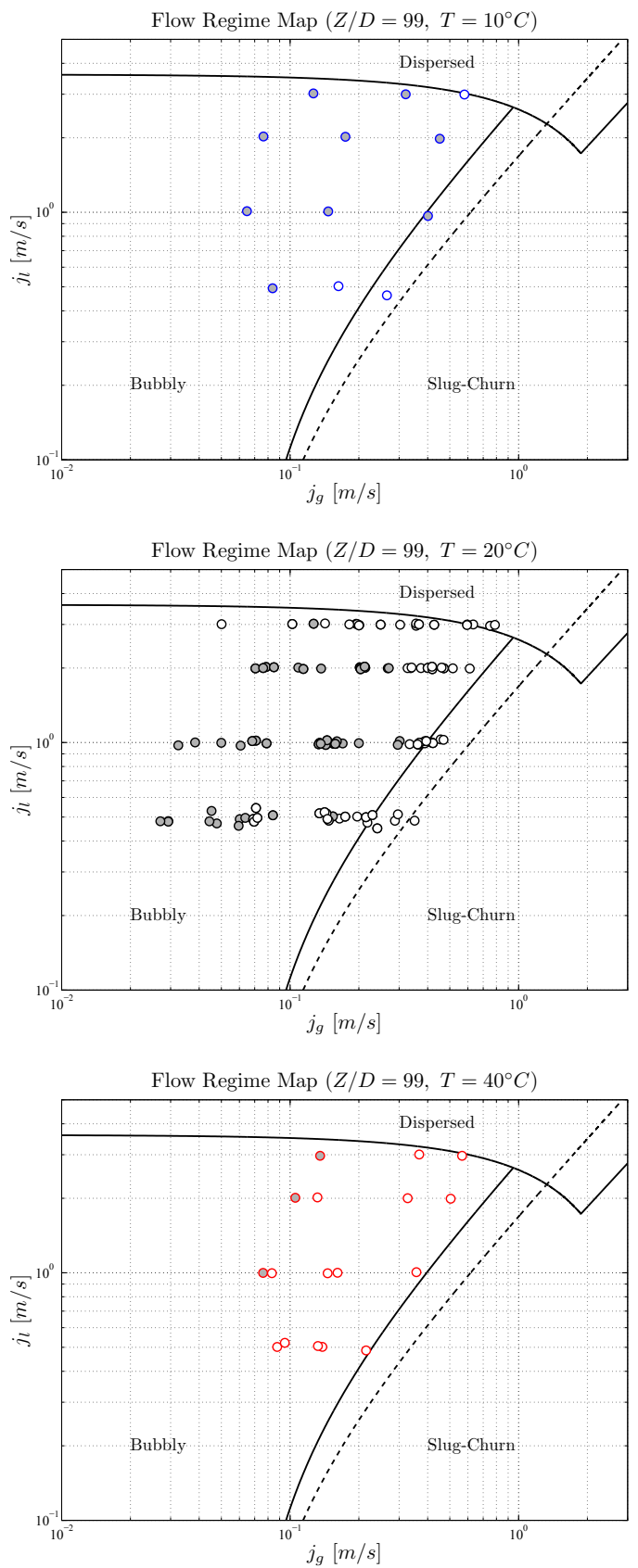

Figure 6: Influence of the temperature on the flow distribution. 

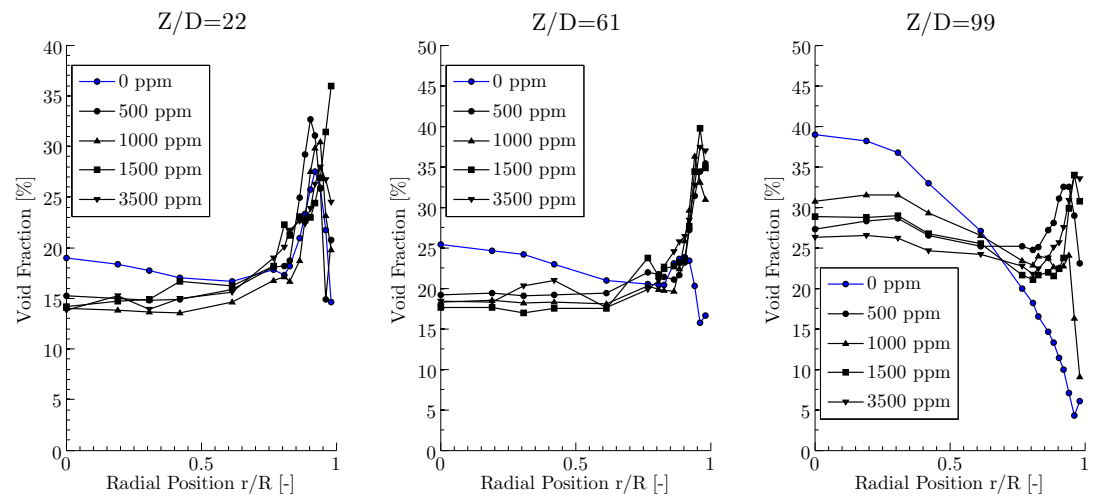

Figure 7: Influence of the electrolyte concentration for $j_{l}=1 \mathrm{~m} / \mathrm{s}$ and $j_{g}=$ $0.35 \mathrm{~m} / \mathrm{s}$ condition.
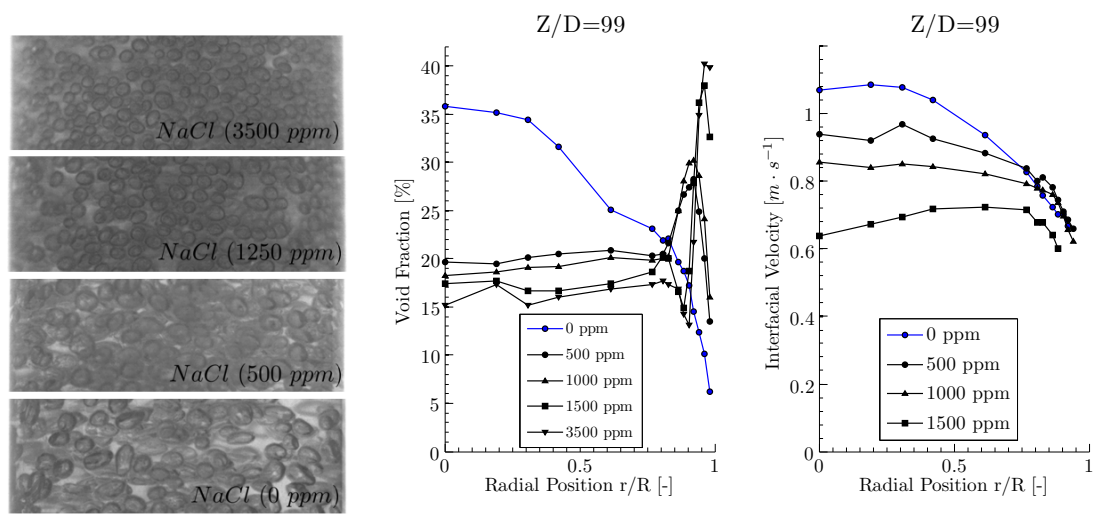

Figure 8: From left to right: Influence of the electrolyte concentration over the bubble size and shape, void fraction profiles and interfacial velocity profiles for $j_{l}=0.5 \mathrm{~m} / \mathrm{s}$ and $j_{g}=0.15 \mathrm{~m} / \mathrm{s}$ condition.

Fig. 9 shows the Taitel map at top location for all electrolyte concentrations tested. This map looks similar to the one in Fig. 3 as the transition criteria used (Equation 1) is binary. Then, no big differences. A map showing all the types of structures would help to notice the changes.

Many authors point out that the addition of electrolytes in polar liquids inhibits the bubble coalescence [21-23]. This effect has a strong influence on the diameter of the bubbles that are generated at the sparger (or any injection system based in a porous medium). By using high-speed photography it can be noticed that the gas flows from the sparger pores and tends to form small bubbles that coalesce. The inhibition of this coalescence results in the formation of smaller bubbles. In 


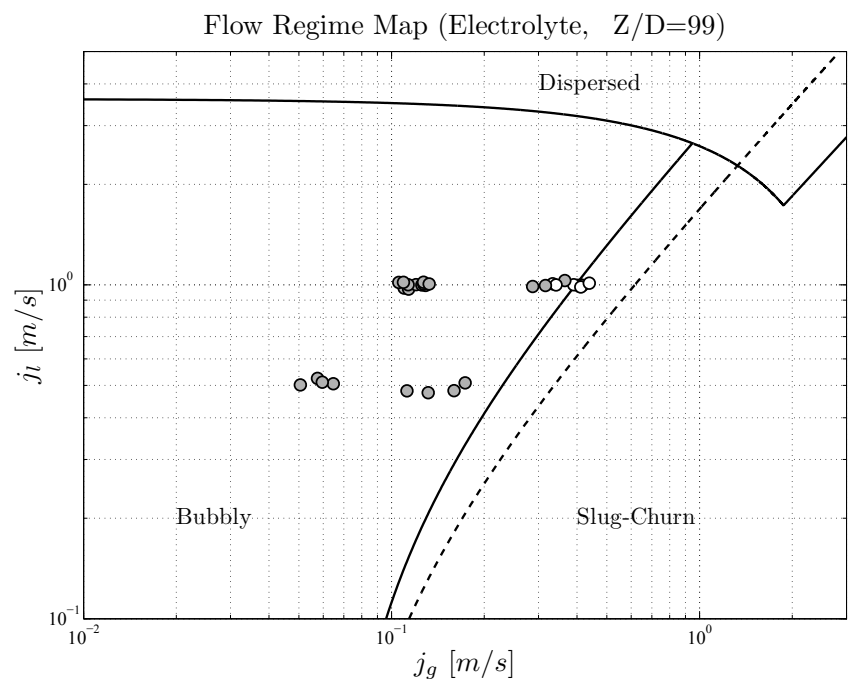

Figure 9: Influence of electrolyte concentration on the Taitel map.

addition, this inhibition leads to a delay in the transition to core distributions thus influencing the axial development of the flow structure.

\section{Conclusions}

In this work we have demonstrated the strong influence of both temperature changes and electrolyte concentration on the flow configuration and its axial development in vertical pipe flows. Interestingly, these effects are not predicted by present correlations and prediction tools such as the Taitel map. In particular, the effect of electrolyte concentration is not taken into account in present coalescence models employed for the Eulerian-Eulerian simulation of two-phase flow. Thus, the control of system temperature and water quality is mandatory for the development of an accurate database. Also, a generalization of the Taitel map able to predict such changes is also needed.

\section{Acknowledgements}

The authors sincerely thank the Spanish Ministerio de Economía y Competitividad for funding the project ENE2013-48565-C2-2-P.

\section{References}

[1] Ishii, M. \& Hibiki, T., Thermo-fluid dynamics of two-phase flow. Springer, 2006. 
[2] Todreas, N.E. \& Kazimi, M.S., Nuclear systems, volume 1. CRC Press, 2010.

[3] Brummer, R., Rheology essentials of cosmetic and food emulsions. Springer, 2006.

[4] Mueller, J., Boyle, W.C. \& Popel, H.J., Aeration: principles and practice, volume 11. CRC Press, 2002.

[5] J. Climent, R. Martínez-Cuenca, J.G. Berlanga, B. Julián-Liópez \& S. Chiva, CFD Simulation Of Hydrodynamics And Biological Reactions In An Activated Sludge Process Of WWTP. Proc. of the World Water Congress \& Exhibition (IWA 2014), Lisboa, 2014.

[6] Hibiki, T., Hogsett, S. \& Ishii, M., Local measurement of interfacial area, interfacial velocity and liquid turbulence in two-phase flow. Nuclear Engineering and Design, 184(2), pp. 287-304, 1998.

[7] Ohnuki, A. \& Akimoto, H., Experimental study on transition of flow pattern and phase distribution in upward air-water two-phase flow along a large vertical pipe. International Journal of Multiphase Flow, 26(3), pp. 367-386, 2000.

[8] Hibiki, T. \& Ishii, M., Interfacial area concentration in steady fully-developed bubbly flow. International Journal of Heat and Mass Transfer, 44(18), pp. 3443-3461, 2001.

[9] Lucas, D., Beyer, M. \& Szalinski, L., Experimental database on steam-water flow with phase transfer in a vertical pipe. Nuclear Engineering and Design, 265, pp. 1113-1123, 2013.

[10] Prasser, H.M., Beyer, M., Carl, H., Gregor, S., Lucas, D., Pietruske, H., Schütz, P. \& Weiss, F.P., Evolution of the structure of a gas-liquid two-phase flow in a large vertical pipe. Nuclear Engineering and Design, 237(15), pp. 1848-1861, 2007.

[11] Jochen Zschau, Winfried Zippe, Cornelius Zippe, Horst-Michael Prasser, Dirk Lucas, Ulrich Rohde, Arnd Böttger, Peter Schütz, Eckhard Krepper, Frank-Peter Weiß, et al., Strömungskarten und Modelle für transiente Zweiphasenströmungen. Forschungszentrum Rossendorf, Germany, Report No FZR-379, p. 183, 2003.

[12] Cheng, L., Frontiers and Progress in Multiphase Flow I. Springer, 2014.

[13] Hernández-Pérez, V., Abdulkadir, M. \& Azzopardi, B., Slugging frequency correlation for inclined gas-liquid flow. World Academy of Science, Engineering and Technology, 6, pp. 44-51, 2010.

[14] Escrig, J., Hewakandamby, B., Belt, R. \& Baptista, R., Influence of the gas injection method on the development of two-phase flow in vertical pipes. Proc. Multiphase Flow Conference in Dresden-Rossendorf 2014, Dresden, 2014.

[15] Hibiki, T. \& Ishii, M., Effect of inlet geometry on hot-leg U-bend two-phase natural circulation in a loop with a large diameter pipe. Nuclear Engineering and Design, 203(2), pp. 209-228, 2001.

[16] Taitel, Y., Bornea, D. \& Dukler, A., Modelling flow pattern transitions for steady upward gas-liquid flow in vertical tubes. AIChE Journal, 26(3), pp. 345-354, 1980. 
[17] Kaichiro, M. \& Ishii, M., Flow regime transition criteria for upward twophase flow in vertical tubes. International Journal of Heat and Mass Transfer, 27(5), pp. 723-737, 1984.

[18] Clift, R., Grace, J.R. \& Weber, M.E., Bubbles, drops, and particles. Courier Dover Publications, 2005.

[19] Tomiyama, A., Kataoka, I., Zun, I. \& Sakaguchi, T., Drag coefficients of single bubbles under normal and micro gravity conditions. JSME International Journal Series B, Fluids and Thermal Engineering, 41(2), pp. 472-479, 1998.

[20] Tomiyama, A., Tamai, H., Zun, I. \& Hosokawa, S., Transverse migration of single bubbles in simple shear flows. Chemical Engineering Science, 57(11), pp. 1849-1858, 2002.

[21] Kazakis, N., A, M. \& Paras, S., Experimental study of bubble formation at metal porous spargers: Effect of liquid properties and sparger characteristics on the initial bubble size distribution. Chemical Engineering Journal, 137(2), pp. 265-281, 2008.

[22] Craig, V., Ninham, B. \& Pashley, R., Effect of electrolytes on bubble coalescence. Nature, 364(6435), pp. 317-319, 1993.

[23] Craig, V.S., Bubble coalescence and specific-ion effects. Current Opinion in Colloid \& Interface Science, 9(1-2), pp. 178-184, 2004. 